\title{
Oral Administration of Poly-Gamma-Glutamic Acid Significantly Enhances the Antitumor Effect of HPV16 E7-Expressing Lactobacillus casei in a TC-1 Mouse Model
}

\author{
Eunjin Kim ${ }^{1,2}$, Jihyun Yang ${ }^{1}$, Moon-Hee Sung ${ }^{3,4}$, and Haryoung Poo ${ }^{1 *}$ \\ ${ }^{1}$ Infectious Disease Research Center, Korea Research Institute of Bioscience and Biotechnology, Daejeon 34141, Republic of Korea \\ ${ }^{2}$ College of Veterinary Medicine, Chungnam National University, Daejeon 34134, Republic of Korea \\ ${ }^{3}$ Department of Bio and Fermentation Convergence Technology, Kookmin University, Seoul 02707, Republic of Korea \\ ${ }^{4}$ Bioleaders Corporation, Youngin 16827, Republic of Korea
}

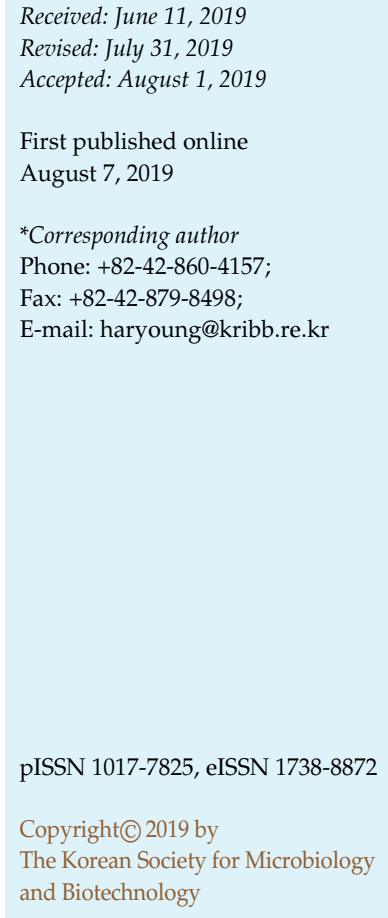

The conventional prophylactic vaccines for human papillomavirus (HPV) efficiently prevent infection with high-risk HPV types, but they do not promote therapeutic effects against cervical cancer. Previously, we developed HPV16 E7-expressing Lactobacillus casei (L. casei-E7) as a therapeutic vaccine candidate for cervical cancer, which induces antitumor therapeutic effects in a TC-1 murine cancer model. To improve the therapeutic effect of L. casei-E7, we performed co-treatment with poly-gamma-glutamic acid ( $\gamma$-PGA), a safe and edible biomaterial naturally secreted by Bacillus subtilis. We investigated their synergistic effect to improve antitumor efficacy in a murine cancer model. The treatment with $\gamma$-PGA did not show in vitro cytotoxicity against TC-1 tumor cells; however, an enhanced innate immune response including activation of dendritic cells was observed. Mice co-administered with $\gamma$-PGA and L. casei-E7 showed significantly suppressed growth of TC-1 tumor cells and an increased survival rate in TC-1 mouse models compared to those of mice vaccinated with L. casei-E7 alone. The administration of $\gamma$-PGA markedly enhanced the activation of natural killer (NK) cells but did not increase the E7-specific cytolytic activity of $\mathrm{CD}^{+} \mathrm{T}$ lymphocytes in mice vaccinated with $L$. casei-E7. Overall, our results suggest that oral administration of $\gamma$-PGA induces a synergistic antitumor effect in combination with L. casei-E7.

Keywords: Poly-gamma-glutamic acid, adjuvant, Lactobacillus casei, HPV16 E7, cervical cancer, natural killer cells

\section{Introduction}

Persistent infection with human papillomavirus (HPV) is associated with cervical cancer, which is the second leading cause of cancer-related deaths among women [1, 2]. To date, more than 40 subtypes of HPVs that infect human genitals have been reported, and of them, HPV type 16 (HPV16) is found in 50\% of cervical cancers [3-6]. Although prophylactic vaccines are used worldwide for the prevention of several types of HPV infection, they barely induce therapeutic effects against persistent infections and the precancerous stage $[3,7]$. Thus, clinical studies of several therapeutic vaccines have been investigated in patients with no effective vaccine efficacy [8]. As the E7 protein is expressed in cervical cancer cells, HPV16 E7 is considered a potential target antigen for therapeutic vaccines [9, 10]. Effective therapeutic cancer vaccines induce robust immunogenicity against tumor antigens, such as activation of cytotoxic T lymphocytes (CTLs) and natural killer (NK) cells [8]. Targeting HPV16 E7 has been reported to eradicate $\mathrm{HPV}$-infected cells by inducing antigen-specific cytotoxic $\mathrm{CD}^{+} \mathrm{T}$ cell responses [8, 11]. Recently, we developed Lactobacillus casei expressing HPV16 E7 protein on its surface (L. casei-E7) using a surface display system. Upon oral administration of L. casei-E7 in a mouse model, we found that it significantly enhanced E7- 
specific serum immunoglobulin G (IgG) and mucosal IgA levels. Additionally, it significantly induced cellular immune responses by enhancing E7-specific lymphocytes and interferon- $\gamma$ (IFN- $\gamma$ )-secreting cells in splenocytes. In HPV16 E7-expressing TC-1 tumor-bearing mice, oral administration of L. casei-E7 was observed to significantly reduce TC-1 tumor size and remarkably increase the survival rate of immunized mice compared to those of the control group (L. casei) mice [4].

Poly-gamma-glutamic acid ( $\gamma$-PGA) is naturally secreted by Bacillus subtilis and composed of glutamic acid polymers bonded via $\gamma$-amide linkages $[12,13]$. As $\gamma$-PGA is an ingredient of fermented soybean foods, its oral uptake is considered safe $[13,14]$. Specifically, $\gamma$-PGA has been reported to promote activation of dendritic cells (DCs), leading to an increase in T helper cell type 1 (Th1)-mediated immune responses [15]. Moreover, oral administration of $\gamma$ PGA has been shown to induce antitumor effects by stimulating the activation of NK cells. These findings imply that $\gamma$-PGA can have a synergistic effect on the antitumor effect of L. casei-E7 [13, 15, 16].

In this study, we investigated the synergistic antitumor effect of $\gamma$-PGA on L. casei-E7 vaccination. Compared to those of mice that were orally administered with L. casei or L. casei-E7 alone, the tumor size and survival rate of mice co-administered with $\gamma$-PGA and L. casei-E7 were observed to be alleviated in TC-1 tumor-bearing mice. To understand the mechanism of antitumor activity, E7-specific cytolytic T cell frequency was analyzed using HPV16 E7 ${ }_{49-57}$ peptide (RAHYNIVTF) presented with $\mathrm{H}-2 \mathrm{D}^{\mathrm{b}}$ MHC I Tetramer. Additionally, IFN- $\gamma$-secreting cells were evaluated in the spleen by flow cytometry and enzyme-linked immunospot (ELISPOT) assay. NK cell activation was analyzed by investigating the surface expression of lysosomal-associated membrane protein 1 (CD107a), a functional marker to study NK cell activation. Our results revealed that oral treatment with $\gamma$-PGA enhanced the activities of CD107aexpressing NK cells but it did not increase the E7-specific cytolytic $\mathrm{T}$ cells in mice administered with L. casei-E7. Overall, our results showed that $\gamma$-PGA markedly enhanced the antitumor efficacy of L. casei-E7 vaccine. Thus, $\gamma$-PGA may be a promising adjuvant for $L$. casei-E7 vaccine and other therapeutic tumor vaccines.

\section{Materials and Methods}

\section{Mice, Cells, and Reagents}

Six-week-old female C57BL/6 mice were purchased from Orient Bio Inc. (Korea). Mice were housed in the specific pathogen-free animal facility at the Korea Research Institute of Bioscience and Biotechnology (KRIBB, Korea). All animal experiments were reviewed and approved by the Institutional Animal Care and Use Committee (IACUC) of KRIBB and experiments were performed according to the approved guidelines (KRIBB-AEC-16154, KRIBBAEC-17036). The primary lung epithelial cells of C57BL/6 mice, TC-1 cells, were transduced with retroviral vectors expressing HPV16 E6/E7 and activated c-Ha-ras, which were kindly provided by TC Wu (Johns Hopkins University, USA). RAW 264.7 cells, mouse macrophage cell line, were purchased from American Type Culture Collection (ATCC, USA). TC-1 cells and RAW 264.7 cells were maintained in RPMI 1640 (Gibco-BRL, USA) containing $10 \%$ heat-inactivated fetal bovine serum (FBS; Gibco-BRL, USA), $100 \mathrm{U} / \mathrm{ml}$ penicillin, and $100 \mathrm{mg} / \mathrm{ml}$ streptomycin (Gibco-BRL). To generate bone marrow-derived dendritic cells (BMDCs), bone marrow cells were incubated with $20 \mathrm{ng} / \mathrm{ml}$ granulocytemacrophage colony-stimulating factor (GM-CSF) and $10 \mathrm{ng} / \mathrm{ml}$ interleukin 4 (IL-4) (Peprotech, USA) for 7 days, with $50 \%$ of media being changed on days 3 and 6 by substituting half of the media containing GM-CSF and IL-4. L. casei-E7 $\left(5 \times 10^{9}\right.$ cells/mouse) was provided by Bioleaders Corporation (Korea), and $\gamma$-PGA (average molecular mass $=2,000 \mathrm{kDa}$ ) was purchased from Bioleaders Corporation (Korea).

\section{Viability Assay}

TC- 1 cells $\left(2 \times 10^{3}\right.$ cells/well $)$ were seeded in a 96-well culture plate and treated with 0 to $0.5 \mathrm{mg} / \mathrm{ml} \gamma$-PGA or $0.5 \%$ Triton X-100 as a positive control for $48 \mathrm{~h}$. After the treatment, $20 \mu \mathrm{l}$ of WST-1 solution (Roche, Switzerland) was added to each well and incubated for an additional $2 \mathrm{~h}$. Subsequently, the optical density (OD) was measured at $420 \mathrm{~nm}$ using a microplate reader (Molecular Devices, USA), and the OD of PBS-treated cells (control) was considered the $100 \%$ survival rate for comparative analysis.

\section{Enzyme-Linked Immunosorbent Assay (ELISA)}

RAW 264.7 cells were incubated with $\gamma$-PGA $(200 \mu \mathrm{g} / \mathrm{ml})$ or lipopolysccharide (LPS) $(1 \mu \mathrm{g} / \mathrm{ml})$ for $24 \mathrm{~h}$. Culture supernatants were harvested and assessed to determine tumor necrosis factor- $\alpha$ (TNF- $\alpha$ ) levels using an OptEIA kit (BD Biosciences, USA) according to the manufacturer's instructions. Briefly, an anti-TNF$\alpha$ antibody-coated plate was blocked with $10 \%$ FBS in PBS, and cultured supernatants were incubated for $2 \mathrm{~h}$. Then, biotinylated anti-mouse TNF and streptavidin-horseradish peroxidase conjugate were added for $1 \mathrm{~h}$. The plate was developed with a chromogenic tetramethylbenzidine substrate (BD Biosciences, USA), and each well was terminated with stop solution $\left(2 \mathrm{~N} \mathrm{H}_{2} \mathrm{SO}_{4}\right)$. The OD was measured at $450 \mathrm{~nm}$ using a microplate reader.

\section{Oral Administration and Tumor Challenge}

TC- 1 cells at a seeding density of $2 \times 10^{4}$ cells in $100 \mu$ of PBS were subcutaneously injected on the right-side flank of each C57BL/ 6 mouse ( $n=10$ per group). To investigate the therapeutic efficacy of $\gamma$-PGA $(2,000 \mathrm{kDa} ; 800 \mu \mathrm{g} /$ mouse) and L. casei-E7 $(5 \times$ 
$10^{9}$ cells/mouse), oral administration was initiated one week after tumor cell challenge. Mice were administered $800 \mu \mathrm{g}$ of $\gamma$-PGA $(2,000 \mathrm{kDa})$ or PBS five times per week on weeks $0,3,5,6$, and 7 via the intragastric route. Oral administration of L. casei $\left(5 \times 10^{9}\right.$ cells/mouse) or L. casei-E7 ( $5 \times 10^{9}$ cells/mouse) was supplied five times per week on weeks $1,2,4$, and 8 . Tumor size was measured three times per week using a digital caliper, and tumor volume was calculated according to the formula: volume in $\mathrm{mm}^{3}=(\mathrm{L} \times$ $\left.\mathrm{W}^{2}\right) / 2$, where the two-dimensional longest axis is denoted by $\mathrm{L}$ $(\mathrm{mm})$ and the shortest axis by $\mathrm{W}(\mathrm{mm})$.

\section{Flow Cytometry}

To evaluate the effect of $\gamma$-PGA on cytotoxicity, TC- 1 cells $(5 \times$ $10^{5}$ cells/well) were treated with $\gamma$-PGA $(0,0.0625,0.125,0.25$, and $0.5 \mathrm{mg} / \mathrm{ml})$ or puromycin $(1 \mu \mathrm{g} / \mathrm{ml}$; Sigma Aldrich, USA) and incubated for $48 \mathrm{~h}$. After incubation, the cells were harvested and stained using a 7-aminoactinomycin D (7-AAD)/Annexin V staining kit (BD Biosciences, USA). To determine the expression of surface markers of BMDCs, cells were treated with $\gamma$-PGA $(200 \mu \mathrm{g} / \mathrm{ml})$ or LPS $(1 \mu \mathrm{g} / \mathrm{ml})$ for $24 \mathrm{~h}$ and then stained with fluorescent dye-conjugated anti-mouse clusters of differentiation 40 (CD40), CD80, CD86, and MHC class II (MHC II) antibodies (BD Biosciences). To investigate the frequency of CD107aexpressing NK cells, splenocytes $\left(5 \times 10^{5}\right.$ cells $)$ were harvested from the administered mice and co-cultured with TC- 1 cells $(5 \times$ $10^{4}$ cells) in the presence of Golgiplug (BD Bioscience) for $3 \mathrm{~h}$, followed by further incubation with PE-conjugated anti-mouse CD107a antibodies ( $1 \mu \mathrm{l} /$ sample; BD Biosciences) for $12 \mathrm{~h}$. Furthermore, cells were incubated with anti-mouse CD16/CD32 antibodies (BD Biosciences) for blocking Fc $\gamma$ receptors and stained with PerCP-conjugated anti-mouse $\mathrm{T}$ cell surface glycoprotein CD3 epsilon chain (CD3ع) (BD Biosciences) and Alexa 647conjugated anti-mouse killer cell lectin-like receptor subfamily $B$ member 1C (NK1.1) antibodies (Biolegend, USA). To evaluate the percent of antigen-specific $\mathrm{CD} 8^{+} \mathrm{T}$ cells, splenocytes $\left(1 \times 10^{6}\right.$ cells $)$ were stained with APC-conjugated HPV16 E7 49-57 peptide (RAHYNIVTF) presented with $\mathrm{H}-2 \mathrm{D}^{\mathrm{b}}$ MHC I tetramer (MBL, USA) and FITC-conjugated anti-mouse CD8 antibodies (BD Biosciences) for $30 \mathrm{~min}$. The stained cells were evaluated using a FACSCalibur flow cytometer with CellQuest Pro software (BD Biosciences), and data were analyzed by FlowJo software (Tree Star, USA).

\section{Enzyme-Linked Immunospot (ELISPOT) Assay}

Splenocytes from the orally administered mice $\left(5 \times 10^{5}\right.$ cells $/$

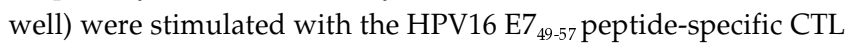
peptide (RAHYNIVTF) $(1 \mu \mathrm{g} / \mathrm{ml}$; AnaSpec Inc., USA) for $72 \mathrm{~h}$. The number of IFN- $\gamma$-producing spot-forming units (SFUs) was measured using a mouse IFN- $\gamma$ ELISPOT kit (BD Biosciences) according to the manufacturer's instructions.

\section{Statistical Analysis}

Statistical differences between the two groups were assessed using two-tailed Student's $t$-test, and one-way ANOVA followed by Bonferroni correction (ANOVA/Bonferroni) to compare multiple groups. All data are presented as the mean \pm standard deviation (SD) except for tumor size, survival rate, and tumor-free mouse rate, which are shown as the mean \pm standard error of the mean (SEM). The log-rank test was used to analyze the survival rate and tumor-free mouse rate between the two groups. Tumor size, survival rate, and tumor-free mouse rate analyses were performed using Prism software (GraphPad, USA). $p<0.05$ was considered a statistically significant difference.

\section{Results}

Treatment with $\gamma$-PGA Does Not Affect the Viability of Tumor Cells but Induces the Activation of Innate Immune Cells In Vitro

To investigate whether $\gamma$-PGA directly affects cytotoxicity of tumor cells, we first examined the effect of $\gamma$-PGA on the viability of tumor cells. TC- 1 cells were treated with $\gamma$-PGA $(2,000 \mathrm{kDa} ; 0-0.5 \mathrm{mg} / \mathrm{ml})$ for $48 \mathrm{~h}$. Furthermore, cell viability and cell death were analyzed by WST-1 assay and Annexin-V staining, respectively. As shown in Fig. 1A, treatment with $\gamma$-PGA did not affect the viability of TC-1 cells $(97.58 \pm 1.24 \%)$, while treatment with Triton X-100 (control) was observed to significantly decrease the percent of viability $(6.81 \pm 2.52 \%)$. Further analysis revealed that the number of apoptotic (Annexin- $\mathrm{V}^{+}$) and necrotic (7$\mathrm{AAD}^{+} /$Annexin- $\mathrm{V}^{-}$) cells was lower after treatment with $\gamma$-PGA $(9.35 \pm 1.53 \%)$. However, upon treatment with puromycin as a control, a considerable increase in the percent of apoptotic and necrotic cells $(46.77 \pm 10.99 \%)$ was observed (Fig. 1B). Overall, the results suggested that $\gamma$-PGA does not induce cytotoxicity in tumor cells. In vitro treatment of $\gamma$-PGA has been reported to induce innate immune responses, such as activation and cytokine production of macrophage and DCs [13-15]. Thus, we evaluated the effect of $\gamma$-PGA on innate immune responses using RAW 264.7 cells (macrophage cells) and BMDCs. RAW 264.7 cells were incubated with $\gamma$-PGA $(200 \mu \mathrm{g} / \mathrm{ml})$ or LPS $(1 \mu \mathrm{g} / \mathrm{ml})$ for $24 \mathrm{~h}$, and the culture supernatants were then assessed by ELISA to determine the expression level of TNF- $\alpha$. As shown in Fig. 1C, $\gamma$-PGA induced 13.5-fold higher TNF- $\alpha$ expression levels than PBS treatment. A similar trend was observed with TNF- $\alpha$ expression levels upon LPS treatment $(p<0.001)$ secreted from RAW 264.7 cells compared to TNF- $\alpha$ levels upon PBS treatment. Furthermore, the expression levels of co-stimulatory molecules, such as CD40, CD80, CD86, and MHC II molecules, were significantly increased in $\gamma$-PGA-treated BMDCs compared to their expression levels in PBS-treated 
A
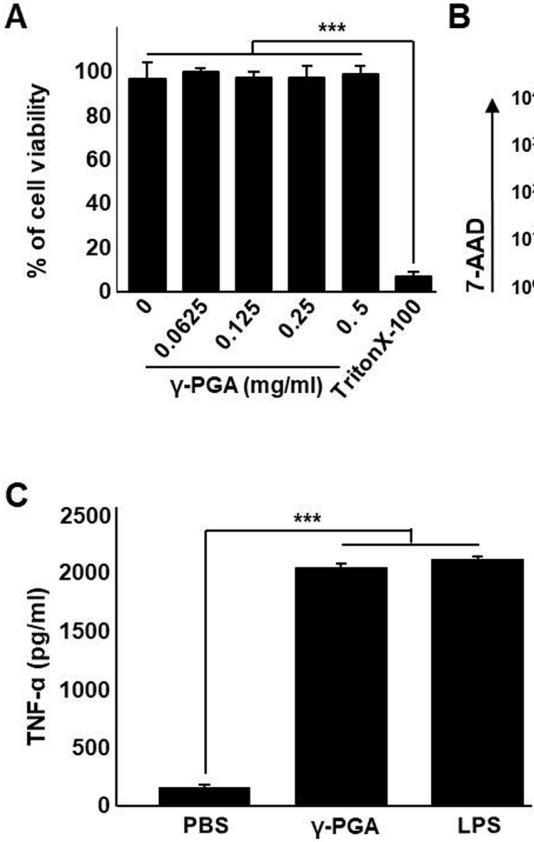

B

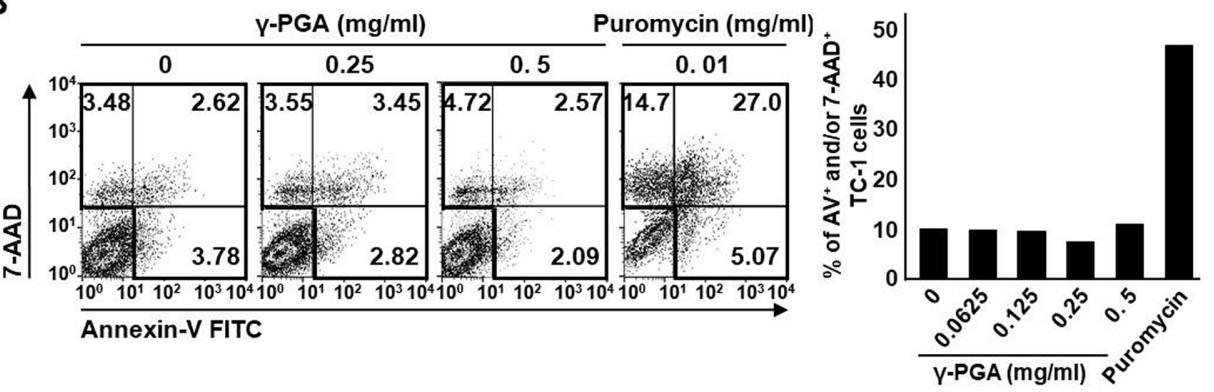

D

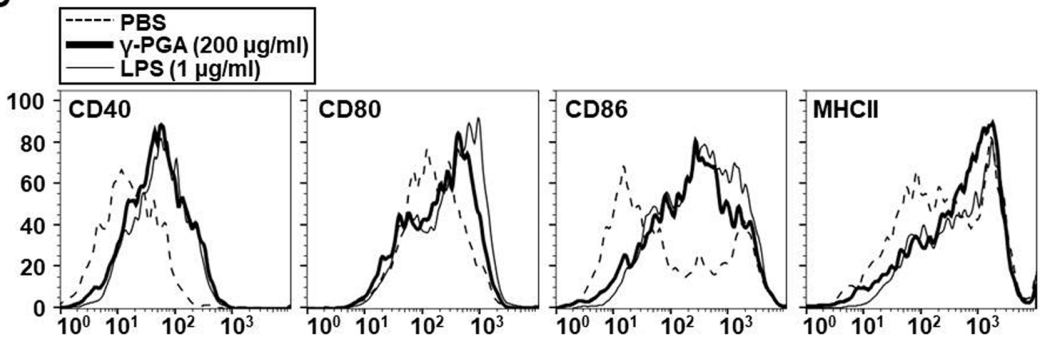

Fig. 1. The effect of $\gamma$-PGA on the viability of tumor cells and the activation of immune cells in vitro.

(A) TC-1 cells were treated with 0 to $0.5 \mathrm{mg} / \mathrm{ml} \gamma$-PGA or $0.5 \%$ Triton X-100 for $48 \mathrm{~h}$. To determine the viability of tumor cells, TC-1 cells were subjected to a WST-1 assay. (B) To measure the percentages of apoptotic and necrotic cells, TC-1 cells were treated with 0 to $0.5 \mathrm{mg} / \mathrm{ml} \gamma$-PGA or $1 \mu \mathrm{g} / \mathrm{ml}$ puromycin for $48 \mathrm{~h}$, stained using a 7-AAD/Annexin V staining kit, and analyzed by flow cytometry. (C) RAW 264.7 cells were treated with $\gamma$-PGA $(200 \mu \mathrm{g} / \mathrm{ml})$ or LPS $(1 \mu \mathrm{g} / \mathrm{ml})$ for $24 \mathrm{~h}$. The level of TNF- $\alpha$ was measured in the culture supernatants by ELISA. (D) BMDCs were treated with $\gamma$-PGA $(200 \mu \mathrm{g} / \mathrm{ml})$ or LPS $(1 \mu \mathrm{g} / \mathrm{ml})$ for $24 \mathrm{~h}$. The expression of co-stimulatory molecules and MHC II was analyzed by flow cytometry. Statistical differences were analyzed by $t$-test; ${ }^{* * *} p<0.001$ compared with an untreated control. Data are presented as the mean \pm SD and are representative of three independent experiments with similar results.

cells (Fig. 1D). As hypothesized, our results revealed that $\gamma$-PGA induces innate immunity by activating DCs and TNF- $\alpha$ production in macrophages.

Oral Administration of $\gamma$-PGA Markedly Enhances the Antitumor Activity of Mice Immunized with L. casei-E7

To evaluate the therapeutic antitumor efficacy of coadministration of $\gamma$-PGA and L. casei-E7 in the mouse model, TC-1 cells were subcutaneously injected into mice 7 days before oral administration. Then, mice were administered $\gamma$-PGA $(2,000 \mathrm{kDa} ; 800 \mu \mathrm{g} /$ mouse $)$ five times per week via the intragastric route on weeks $0,3,5,6$, and 7. Oral administration of L. casei-E7 $\left(5 \times 10^{9}\right.$ cells/mouse) was given five times per week on weeks $1,2,4$, and 8 (Fig. 2A). Individual administration of $L$. case $i$ was used as a negative control. The tumor volume and mouse survival rate were measured three times per week for 15 weeks after the first oral administration; the absence of tumors was also recorded. Fifty days after administration of the first dose, the tumor volume was found to be significantly decreased in mice co-treated with $\gamma$-PGA and L. casei-E7 $\left(99.82 \pm 55.10 \mathrm{~mm}^{3}\right)$ compared to that of PBS $(963.84 \pm$ $\left.240.90 \mathrm{~mm}^{3}\right)(p<0.05)$ or L. casei $\left(778.88 \pm 282.35 \mathrm{~mm}^{3}\right)(p<$ 0.05 ) treated mice (Fig. 2B). Additionally, the survival rate was observed to be significantly increased in mice cotreated with $\gamma$-PGA and L. casei-E7 (90\%) compared to the survival rate observed in PBS $(10 \%)(p<0.001)$, L. casei $(30 \%)(p<0.01)$, or L. casei-E7 $(40 \%)(p=0.067)$ treated mice at day 85 post-first dose administration (Fig. 2C). The percentage of tumor-free mice was found to be increased in $\gamma$-PGA plus L. casei-E7-treated mice (70\%) compared to that of PBS $(10 \%)(p<0.01)$, L. casei $(30 \%)(p=0.11)$, or L. casei-E7 $(40 \%)(p=0.24)$ treated mice at day 85 after administration of the first dose (Fig. 2D). Overall, the results suggested that administration of $\gamma$-PGA significantly enhanced the antitumor effect of L. casei-E7 in a TC-1 tumor mouse model.

Oral Administration of $\gamma$-PGA Promotes Activation of NK Cells but Does Not Induce E7-Specific CTL Activity in Mice Immunized with L. casei-E7

An earlier study reported that oral administration of L. casei-E7 induces antitumor effects in a TC-1 tumor- 
A

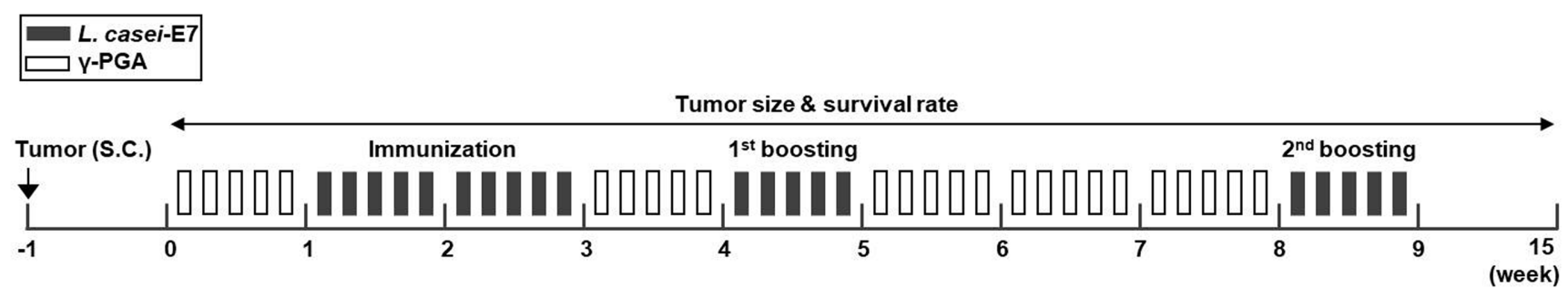

B

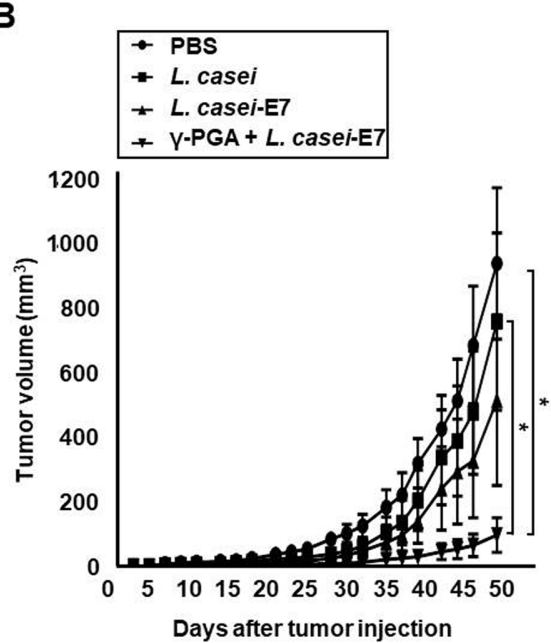

C

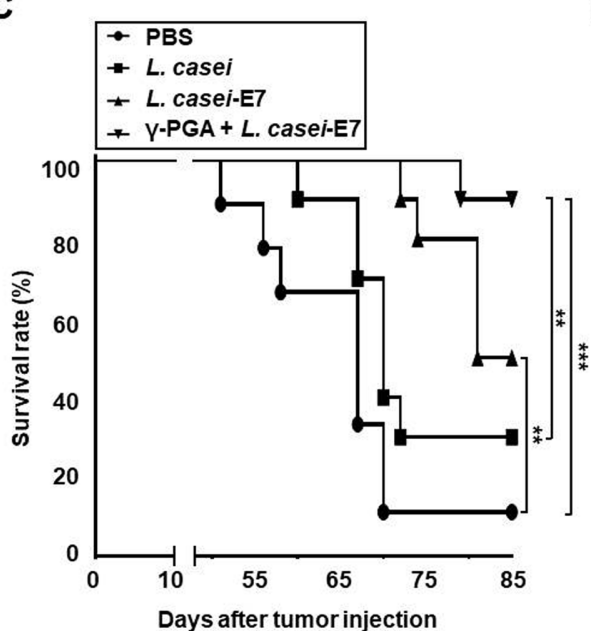

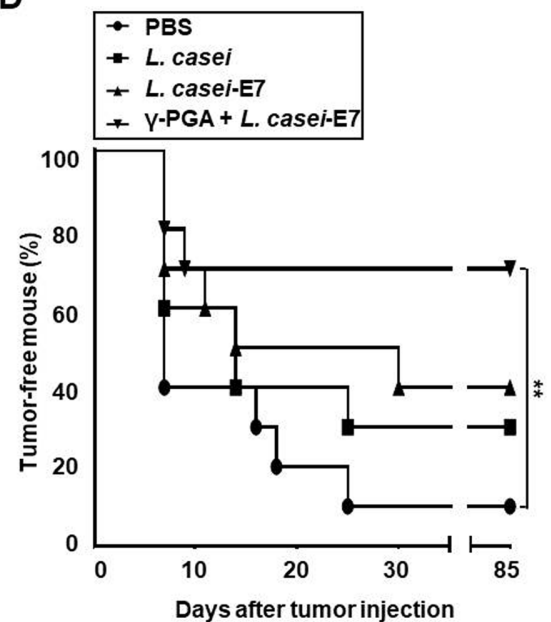

Fig. 2. The antitumor efficacy of oral administration with L. casei-E7 and $\gamma$-PGA.

To investigate the therapeutic efficacy of $L$. casei-E7 and $\gamma$-PGA in tumor-bearing mice, (A) TC- 1 cells $\left(2 \times 10^{4}\right.$ cells $)$ were injected subcutaneously into C57BL/ 6 mice ( $n=10$ /group). One week after tumor cell challenge, mice were orally administered with L. casei-E7 (5 $\times 10^{9}$ cells $\left./ \mathrm{mouse}\right)$ and $\gamma$-PGA (2,000 kDa, $800 \mu \mathrm{g} /$ mouse) five times per week for 9 weeks. (B) Tumor size, (C) survival rate (\%), and (D) tumor-free mice (\%) were monitored three times per week and calculated according to the formula (i.e., $\mathrm{mm}^{3}=\left(\mathrm{L} \times \mathrm{W}^{2}\right) / 2$ (L: the two-dimensional longest axis (in $\left.\mathrm{mm}\right), \mathrm{W}$ : shortest axis (in mm). Statistical differences were analyzed by $t$-test $(\mathbf{B})$ or log-rank test (C and $\mathbf{D}){ }^{*}, p<0.05 ;{ }^{* *}, p<0.01 ;$ and ${ }^{* * *}, p<0.001$. Data are presented as the mean \pm SEM.

bearing mouse model by increasing antigen-specific CTL activity [4]. As $\gamma$-PGA activated innate immune responses capable of enhancing adaptive immunity, we hypothesized that co-treatment of $\gamma$-PGA and L. casei-E7 could upregulate L. casei-E7-mediated CTL activity more than treatment of L. casei-E7 alone. Thus, we performed an experiment as shown in Fig. 3A. Three days after the last administration, splenocytes were isolated and stained with fluorescent dye-conjugated anti-CD8 antibodies and HPV16 E7 ${ }_{49-57^{-}}$ specific Tetramer. As shown in Fig. 3B, flow cytometry revealed that the percentage of $\mathrm{E}_{49-57}$-specific $\mathrm{CD}^{+} \mathrm{T}$ cells significantly increased in $\gamma$-PGA plus L. casei-E7-treated mice $(1.37 \pm 0.36 \%)$ compared to that of PBS $(0.77 \pm 0.18 \%)$ and $L$. casei $(0.81 \pm 0.14 \%)$ treated mice $(p<0.05)$. Compared to $L$. casei-E7 group, $\gamma$-PGA plus $L$. casei-E7 group showed a higher percentage of $\mathrm{E}_{49-57}$-specific $\mathrm{CD}^{+} \mathrm{T}$ cells. However, no significant difference was observed. Furthermore, we found that L. casei-E7 treated mice had a higher percentage of $\mathrm{E7}_{49-57}$-specific CD8 ${ }^{+} \mathrm{T}$ cells $(1.05 \pm 0.26 \%)$ than PBS or L. casei-treated mice. Next, we examined CTL activity by stimulating the splenocytes from the immunized mice with the MHC I-restricted HPV16 E7 49-57 peptide for $72 \mathrm{~h}$. Then, the number of IFN- $\gamma$-producing SFUs was measured via ELISPOT assay. As shown in Fig. 3C, the number of HPV16 $\mathrm{E}_{49-57}$-specific IFN- $\gamma$-producing cells significantly increased approximately 5 -fold in $\gamma$-PGA and L. casei-E7 co-treated mice (43 \pm 5 SFUs) compared to that of PBS ( $5 \pm 2$ SFUs) $(p<0.01)$ or L. casei $(11 \pm 4$ SFUs $)(p<0.01)$ treated mice. Mice treated with L. casei-E7 alone were found to have a significantly increased number of E7-specific IFN- $\gamma$ producing cells ( $43 \pm 8$ SFUs) compared to that of PBS ( $p<$ $0.01)$ or L. casei $(p<0.01)$ treated mice. However, a similar 
A
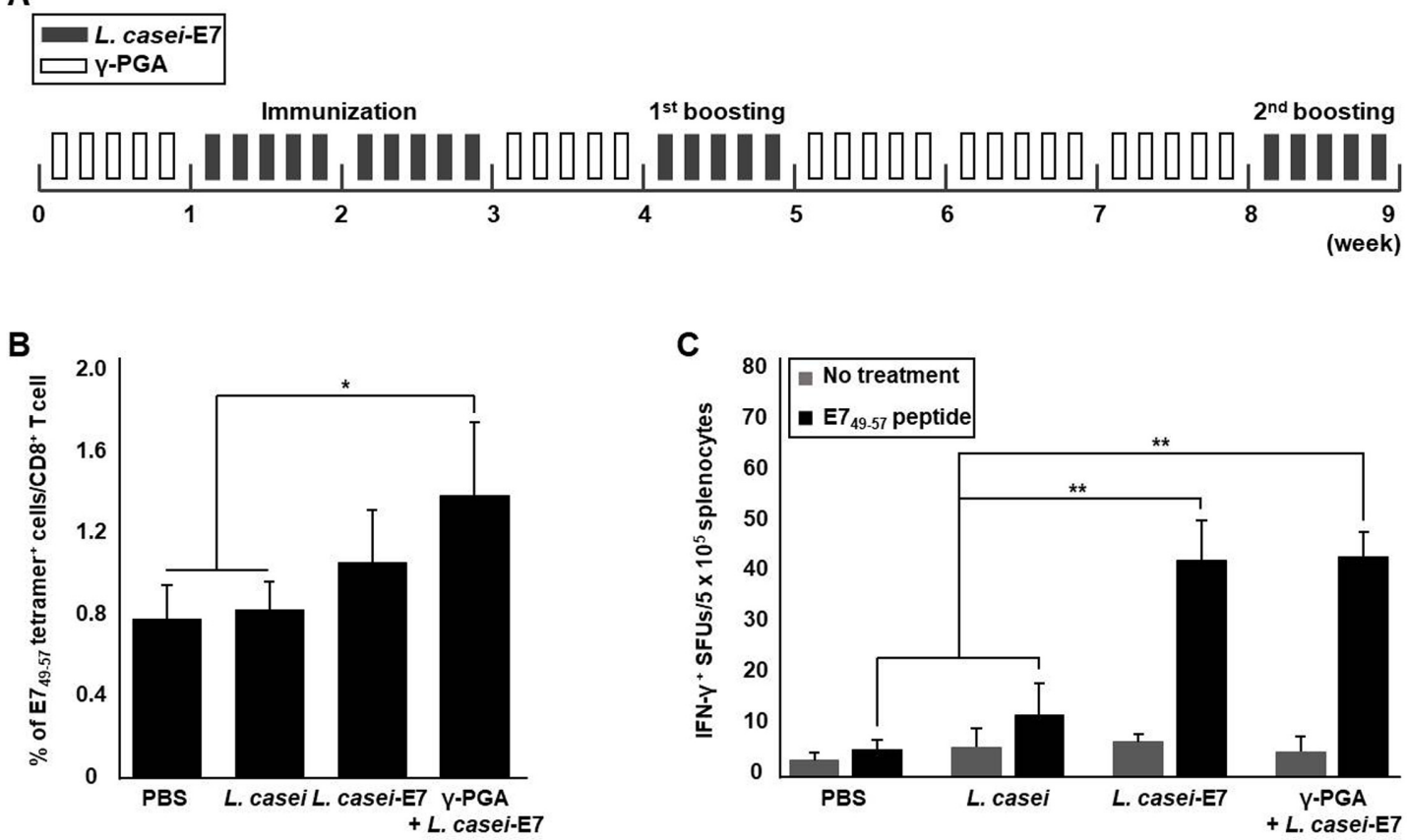

Fig. 3. Antigen-specific CTL responses to oral administration of L. casei-E7 and $\gamma$-PGA.

(A) C57BL/ 6 mice $(n=10)$ were orally given L. casei-E7 $\left(5 \times 10^{9}\right.$ cells $/$ mouse) or $\gamma$-PGA $(2,000 \mathrm{kDa}, 800 \mu \mathrm{g} / \mathrm{mouse})$ five times per week for 9 weeks, and the spleens were harvested from immunized mice. To determine antigen-specific CD8 ${ }^{+} \mathrm{T}$ cells, (B) splenocytes were stained for E7 peptidespecific (H-2D ${ }^{b}$-RAHYNIVTF) CD8 ${ }^{+}$T cells and acquired by flow cytometry, and (C) splenocytes were treated with $1 \mu \mathrm{g} / \mathrm{ml} \mathrm{HPV16} \mathrm{E7-specific}$ CD8-restricted peptide (RAHYNIVTF) for $72 \mathrm{~h}$. Then, the number of IFN- $\gamma$-producing SFUs was measured by IFN- $\gamma$ ELISPOT assay. Statistical differences were analyzed by $t$-test, ${ }^{*}, p<0.05$; and ${ }^{* *}, p<0.01$. Data are presented as the mean \pm SD.

increasing trend was observed in $\gamma$-PGA plus L. casei-E7treated mice. Overall, these results suggested that oral administration of $\gamma$-PGA partially increased L. casei-E7specific CTL activity.

Since NK cells are known as cytolytic effector cells, they play a critical role in the immunosurveillance of tumor cells by directly inducing tumor cell death, particularly when cancer cells evade recognition by $\mathrm{CD}^{+} \mathrm{T}$ cells due to downregulated expression of MHC I molecules [16]. Previously, we reported that oral administration of $\gamma$-PGA enhances the NK cell population [13]. As there was no significant difference observed in induction of CTL activity by oral administration of L. casei-E7 alone and $\gamma$-PGA plus L. casei-E7, we investigated the percentage and activation of NK cells in tumor-bearing mice. Mice were orally administered $\gamma$-PGA and L. casei-E7 as described above, and the frequency of NK cells was analyzed by staining splenocytes with fluorescent dye-conjugated antibodies specific to NK1.1 and CD3e and quantitation via flow cytometry. As shown in Fig. 4A, the percentage of NK1.1 $1^{+}$ $\mathrm{CD} 3 \varepsilon^{-}$cells was significantly increased in mice co-treated with $\gamma$-PGA and L. casei-E7 $(2.55 \pm 0.05 \%)$ compared to that of L. casei $(1.98 \pm 0.11 \%)(p<0.01)$ and L. casei-E7 $(2.22 \pm$ $0.03 \%)(p<0.001)$ treated mice. Thus, the results indicated that co-treatment with $\gamma$-PGA and L. casei-E7 efficiently increased the frequency of NK cells. CD107a is a known functional marker for the activity of NK cells, and its expression correlates with NK cell-mediated lysis of target cells [17]. Subsequently, we investigated the percentage of CD107a-expressing NK cells. As shown in Fig. 4B, the percentage of CD107a-expressing NK cells was higher in $\gamma$ PGA plus L. casei-E7-treated mice $(22.37 \pm 2.33 \%)$ compared to that of L. casei $(18.67 \pm 1.12 \%)(p=0.096)$ and L. casei-E7 $(19.37 \pm 0.64 \%) \quad(p=0.067)$ treated mice. These results suggested that administration of $\gamma$-PGA significantly enhanced the frequency and activation of NK cells induced by L. casei-E7 alone. Overall, our results revealed that $\gamma$-PGA may be a potential adjuvant for a L. casei-E7 therapeutic vaccine to improve the antitumor effect in a TC-1 tumor mouse model and that the mechanism is mediated by NK cell activation. 
A
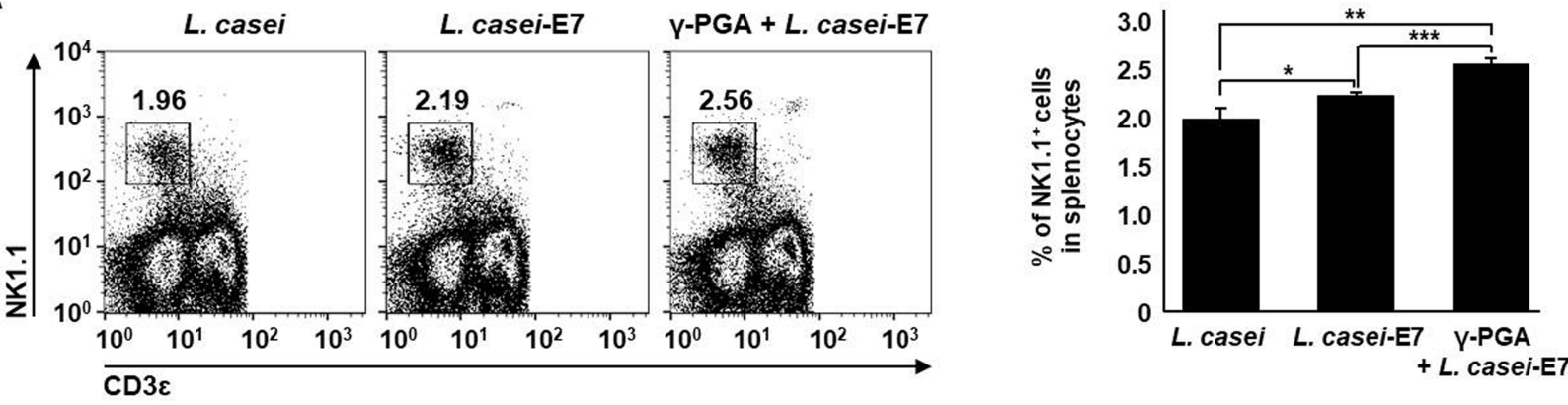

B
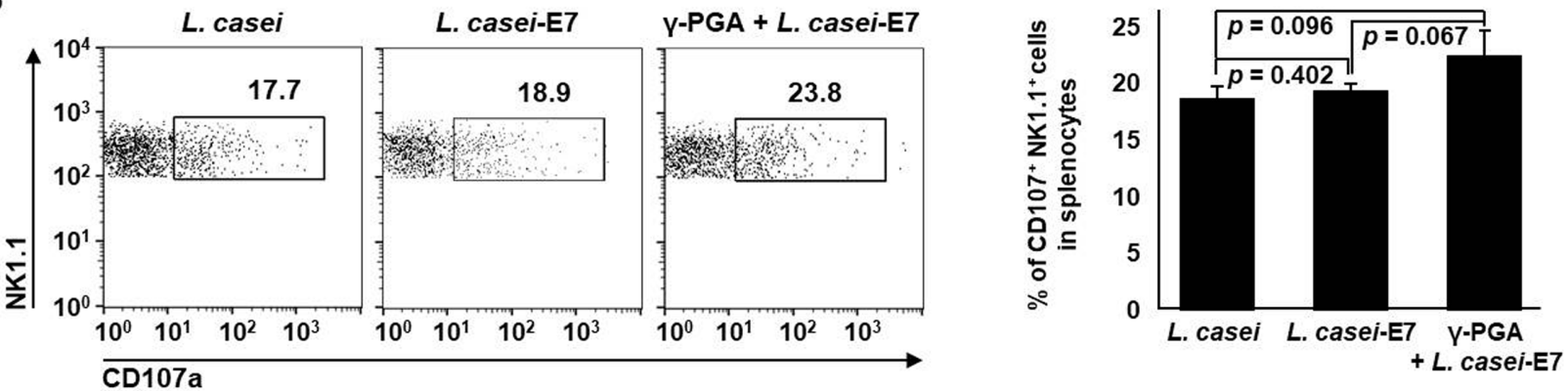

Fig. 4. The increases in NK cells and CD107a-expressing NK cells in response to oral administration of L. casei-E7 and $\gamma$-PGA. (A) To compare the frequencies of NK cells in immunized mice, splenocytes were stained with Alexa 647-conjugated anti-mouse NK1.1 and

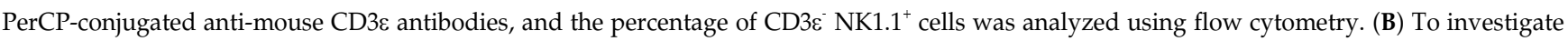
CD107a-expressing $\mathrm{NK}^{+}$cells, splenocytes were co-cultured with TC-1 cells for $3 \mathrm{~h}$ and then cultured with PE-conjugated anti-mouse CD107a antibodies for $12 \mathrm{~h}$. The cells were stained and analyzed by flow cytometry. Statistical differences were analyzed by $t$-test, ${ }^{*}, p<0.05 ; * *, p<0.01$; and ${ }^{* * *}, p<0.001$. Data are presented as the mean \pm SD.

\section{Discussion}

In this study, we showed that oral administration of $\gamma$ PGA significantly enhanced the antitumor efficacy of L. casei-E7 in a TC-1 mouse model. Regression of tumor cell growth and enhanced survival rate was observed for $\gamma$ PGA plus L. casei-E7 treated mice compared to the tumor growth and survival rate for mice immunized with $L$. caseiE7 alone. Furthermore, we showed that the mechanism by which $\gamma$-PGA enhanced the antitumor activity of L. casei-E7 resulted in an increased activation of NK cells rather than an increase in E7-specific CTL activity. NK cells are activated by IL-12 cytokine secreted from innate immune cells (e.g., macrophages and DCs), and we previously reported that $\gamma$-PGA induces the production of IL-12 in macrophages and DCs and increases NK cell activation in both mice and humans. $[13,18]$. Therefore, $\gamma$-PGA-induced IL-12 production may efficiently support NK cell activation. In the case of CTL activity, because L. casei-E7 itself can sufficiently increase antigen-specific CTL activity, combination treatment with $\gamma$-PGA may not induce a significant increase in CTL activity. Several reports have shown that NK cells are crucial in promoting antitumor effects $[13,19]$. In our study, compared to administration of L. casei-E7 alone, co-administration of $\gamma$-PGA and L. casei-E7 showed a significantly increased frequency of NK cells $(p<0.001)$. The expression of the activation marker, CD107a, was increased in co-administration of $\gamma$-PGA and L. casei-E7 compared with that in administration of L. casei-E7 alone $(p=0.067)$. NK cell activation includes increases of cell frequency as well as activation marker expression [20]. When NK cells are activated by tumor antigens, they effectively eliminate the escape of tumor cells in the tumor microenvironment $[19,21]$. In contrast, when NK cells are depleted, enhanced tumor cell growth has been reported $[16,22]$. Notably, in MHC I-downregulated tumors, NK cells are known to be the major compounds of the immune mechanisms that recognize and kill the infected cells and mediate adaptive immune responses [16, 23]. As a result, the development of many cancer vaccines is focused on the activation of NK cells using immunogenic agents [16]. Similarly, our results suggest that oral administration of $\gamma$ - 
PGA significantly enhances the antitumor effect of L. caseiE7, which is mediated via activation of NK cells.

Consistent with previous studies, we found that treatment with $\gamma$-PGA induced innate immunity via increased TNF- $\alpha$ production by macrophages and increased expression of co-stimulatory molecules of DCs [13]. In addition, $\gamma$-PGA itself had no cytotoxicity, similar to a previous report on melanoma [13]. It has been reported that $\gamma$-PGA induces innate immune responses through toll-like receptor 4 (TLR4) signaling that stimulates Th1-biased immune responses $[13,15]$. Thus, as TLR4 signaling promotes innate and Th1-mediated adaptive immune responses, TLR4 can be used to improve the efficacy of cancer vaccines [24]. Various TLR4 agonists have been studied as antitumor immunotherapeutic agents [13, 25], and only monophosphoryl lipid A (MPL) as a TLR4 agonist has been approved for human use [26]. Furthermore, MPL has been investigated as a cancer vaccine adjuvant in many clinical trials $[26,27]$. Thus, we suggest that $\gamma$-PGA can improve the efficacy of vaccines by activating innate immune responses.

Our results revealed the improved antitumor efficacy of L. casei-E7 upon oral administration with $\gamma$-PGA. Administration routes are crucial in determining drug delivery mechanisms and activation of immune responses [28]. Notably, oral administration is the most attractive route due to it being a safe, convenient, and painless method [4, 28]. Furthermore, oral treatment can induce mucosal immune responses that also lead to adaptive immune responses. Several studies have demonstrated that oral administration of antigens and TLR agonist improves overall immune responses $[4,13,28]$. Thus, oral administration of $\gamma$-PGA may be beneficial in improving efficacy. Although prophylactic vaccines have been used worldwide, HPV infection constantly poses a public threat. Because prophylactic HPV vaccines are known to exert no therapeutic effects on pre-existing HPV infections and associated precancerous stages, there is an urgent need to develop a therapeutic vaccine with intense immunogenicity. In this study, oral administration of $\gamma$-PGA remarkably suppressed tumor growth and enhanced the survival rate in TC-1 tumor-bearing mice. Our results showed the synergistic antitumor effect of oral administration of $\gamma$-PGA and L. casei-E7. However, further studies are required to evaluate the therapeutic effect in clinical trials. In conclusion, our study suggests that $\gamma$-PGA via oral administration can be a potent immunotherapeutic adjuvant to cancer vaccines that would induce synergistic antitumor activity against cervical cancer.

\section{Acknowledgments}

This work was supported by the R\&D Convergence Program of the National Research Council of Science \& Technology (NST) of the Republic of Korea (Grant no. CAP-16-02-KIST), and by the National Research Foundation of Korea (NRF) grant funded by the Korea government (Grant no. 2018M3A9H4055203).

\section{Conflict of Interest}

The authors have no financial conflicts of interest to declare.

\section{References}

1. Braaten KP, Laufer MR. 2008. Human Papillomavirus (HPV), HPV-Related Disease, and the HPV Vaccine. Rev. Obstet. Gynecol. 1: 2-10.

2. Steller MA. 2003. Human papillomavirus, it's genes and cancer vaccines. Cancer Cell 3: 7-8.

3. Adachi K, Kawana K, Yokoyama T, Fujii T, Tomio A, Miura $\mathrm{S}$, et al. 2010. Oral immunization with a Lactobacillus casei vaccine expressing human papillomavirus (HPV) type 16 E7 is an effective strategy to induce mucosal cytotoxic lymphocytes against HPV16 E7. Vaccine 28: 2810-2817.

4. Poo H, Pyo HM, Lee TY, Yoon SW, Lee JS, Kim CJ, et al. 2006. Oral administration of human papillomavirus type 16 E7 displayed on Lactobacillus casei induces E7-specific antitumor effects in C57/BL6 mice. Int. J. Cancer 119: 17021709.

5. Munoz N, Bosch FX, de Sanjose S, Herrero R, Castellsague $X$, Shah KV, et al. 2003. Epidemiologic classification of human papillomavirus types associated with cervical cancer. N. Engl. J. Med. 348: 518-527.

6. Lee JS, Poo H, Han DP, Hong SP, Kim K, Cho MW, et al. 2006. Mucosal immunization with surface-displayed severe acute respiratory syndrome coronavirus spike protein on Lactobacillus casei induces neutralizing antibodies in mice. J. Virol. 80: 4079-4087.

7. Ault KA. 2007. Long-term efficacy of human papillomavirus vaccination. Gynecol. Oncol. 107: S27-30.

8. Diniz MO, Lasaro MO, Ertl HC, Ferreira LC. 2010. Immune responses and therapeutic antitumor effects of an experimental DNA vaccine encoding human papillomavirus type 16 oncoproteins genetically fused to herpesvirus glycoprotein D. Clin. Vaccine Immunol. 17: 1576-1583.

9. Leggatt GR, Frazer IH. 2007. HPV vaccines: the beginning of the end for cervical cancer. Curr. Opin. Immunol. 19: 232-238.

10. Narayan S, Choyce A, Linedale R, Saunders NA, Dahler A, Chan E, et al. 2009. Epithelial expression of human papillomavirus type $16 \mathrm{E} 7$ protein results in peripheral CD8 
T-cell suppression mediated by CD4+CD25+ T cells. Eur. J. Immunol. 39: 481-490.

11. Kim JW, Hung CF, Juang J, He L, Kim TW, Armstrong DK, et al. 2004. Comparison of HPV DNA vaccines employing intracellular targeting strategies. Gene Ther. 11: 1011-1018.

12. Zhang H, Zhu J, Zhu X, Cai J, Zhang A, Hong Y, et al. 2012. High-level exogenous glutamic acid-independent production of poly-(gamma-glutamic acid) with organic acid addition in a new isolated Bacillus subtilis C10. Bioresour. Technol. 116: 241-246.

13. Kim DJ, Kim EJ, Lee TY, Won JN, Sung MH, Poo H. 2013. Combination of poly-gamma-glutamate and cyclophosphamide enhanced antitumor efficacy against tumor growth and metastasis in a murine melanoma model. J. Microbiol. Biotechnol. 23: 1339-1346.

14. Poo H, Park C, Kwak MS, Choi DY, Hong SP, Lee IH, et al. 2010. New biological functions and applications of highmolecular-mass poly-gamma-glutamic acid. Chem. Biodivers. 7: 1555-1562.

15. Lee TY, Kim YH, Yoon SW, Choi JC, Yang JM, Kim CJ, et al. 2009. Oral administration of poly-gamma-glutamate induces TLR4- and dendritic cell-dependent antitumor effect. Cancer Immunol. Immunother. 58: 1781-1794.

16. Kim TW, Lee TY, Bae HC, Hahm JH, Kim YH, Park C, et al. 2007. Oral administration of high molecular mass polygamma-glutamate induces NK cell-mediated antitumor immunity. J. Immunol. 179: 775-780.

17. Alter G, Malenfant JM, Altfeld M. 2004. CD107a as a functional marker for the identification of natural killer cell activity. J. Immunol. Methods 294: 15-22.

18. Kim KS, Lee TY, Hong JH, Kim A, Kim SJ, Choi JC, et al. 2013. A single-center, randomized double-blind placebocontrolled study evaluating the effects of poly-gammaglutamate on human NK cell activity after an 8-week oral administration in healthy volunteers. Evid. Based Complement. Alternat. Med. 2013: 635960.

19. Larsen SK, Gao Y, Basse PH. 2014. NK cells in the tumor microenvironment. Crit. Rev. Oncog. 19: 91-105.
20. Koo GC, Peppard JR, Hatzfeld A. 1982. Ontogeny of Nk-1+ natural killer cells. I. Promotion of Nk-1+ cells in fetal, baby, and old mice. J. Immunol. 129: 867-871.

21. Malmberg KJ, Carlsten M, Bjorklund A, Sohlberg E, Bryceson YT, Ljunggren HG. 2017. Natural killer cellmediated immunosurveillance of human cancer. Semin. Immunol. 31: 20-29.

22. Miller GM, Andres ML, Gridley DS. 2003. NK cell depletion results in accelerated tumor growth and attenuates the antitumor effect of total body irradiation. Int. J. Oncol. 23: 1585-1592.

23. Indrova M, Simova J, Bieblova J, Bubenik J, Reinis M. 2011. NK1.1+ cells are important for the development of protective immunity against MHC I-deficient, HPV16-associated tumours. Oncol. Rep. 25: 281-288.

24. Noorpisheh Ghadimi S, Farjadian S, Hatam GR, Kalani M, Sarkari B. 2018. Vaccination with live Attenuated L. major and TLR4 Agonist promotes a Th1 immune response and induces protection against L. major infection in BALB/C Mice. Iran J. Immunol. 15: 74-83.

25. Morefield GL, Hawkins LD, Ishizaka ST, Kissner TL, Ulrich RG. 2007. Synthetic Toll-like receptor 4 agonist enhances vaccine efficacy in an experimental model of toxic shock syndrome. Clin. Vaccine Immunol. 14: 1499-1504.

26. Temizoz B, Kuroda E, Ishii KJ. 2016. Vaccine adjuvants as potential cancer immunotherapeutics. Int. Immunol. 28: 329338.

27. Cluff CW. 2010. Monophosphoryl Lipid A (MPL) as an Adjuvant for Anti-Cancer Vaccines: Clinical Results, pp. 111-123. In Jeannin J-F (ed.), Lipid A in Cancer Therapy, Ed. Springer New York, New York, NY

28. Lee TY, Kim YH, Lee KS, Kim JK, Lee IH, Yang JM, et al. 2010. Human papillomavirus type 16 E6-specific antitumor immunity is induced by oral administration of HPV16 E6expressing Lactobacillus casei in C57BL/6 mice. Cancer Immunol. Immunother. 59: 1727-1737. 